\title{
CAN2PCI: Placa con Interfaz al Bus CAN y PCI con Finalidad Docente
}

\author{
Manuel A. Ortiz, Francisco J. Quiles, Carlos D. Moreno y María Brox \\ Universidad de Córdoba, Escuela Politécnica Superior, Departamento de Arquitectura de \\ Computadores, Electrónica y Tecnología Electrónica, Edificio Leonardo Da Vinci, Campus \\ Universitario de Rabanales, Ctra. Madrid-Cádiz Km. 396-A, 14071 Córdoba-España \\ (e-mail: el1orlom@uco.es)
}

\begin{abstract}
Resumen
En este trabajo se presenta una placa con interfaz al bus CAN y $\mathrm{PCl}$ desarrollada para la utilización en prácticas de las asignaturas relacionadas con redes de control. Se trata de una placa de altas prestaciones pensada para su utilización docente gracias a su facilidad de programación. La placa dispone de dos canales CAN independientes y permite acceso directo a los registros del controlador CAN. Las prácticas tienen como objetivo conocer la red de control en los niveles físico y de enlace y desarrollar un software de conectividad (middleware) que realice la interfaz entre estas capas y la de aplicación de usuario. Se exponen también brevemente las prácticas realizadas en una de las asignaturas donde se imparte redes de control, en la que es fundamental la utilización de un hardware conocido que permita programar las funciones básicas que operan directamente con el controlador de bus CAN.
\end{abstract}

Palabras clave: bus CAN, redes de control, bus $P C l$, finalidad docente

\section{CAN2PCI: Board with Interface to CAN and PCI Bus for Educational Purposes}

\begin{abstract}
In this work the development of a board with interface to the CAN and $\mathrm{PCl}$ bus for its use in lab courses related to control networks, is presented. This board has high benefits and advantages and has been implemented for educational purposes due to its programming facility. The board has two independent CAN channels and it allows direct access to the registers of the CAN controller. The objective of the lab experiments is to study the control networks in the physical and link levels and to develop a middleware that performs the interface between these layers and the user application. The experiments done in one of the courses, which includes control networks, are briefly described. In these practical labs it is very important the use of a known hardware that allows programming the basic functions which directly operate with the CAN bus controller.
\end{abstract}

Keywords: CAN bus, networks control, PCI bus, educational objectives 


\section{INTRODUCCIÓN}

Actualmente se tiende al diseño de sistemas de control distribuidos, y como consecuencia, las redes de control y el intercambio de información a través de ellas cobra una gran importancia. Por este motivo cada vez más se utilizan redes de control estandarizadas, que no solo definen el nivel físico sino que proporcionan un mínimo nivel de enlace que permite la transmisión de datos seguros y tolerante a fallos. Una de estas redes de control es el bus CAN que se ha convertido en el líder como bus serie para aplicaciones empotradas, especialmente en el campo de la automoción. Este bus ha sido elegido para la realización de las prácticas de redes de control en la asignatura de "Arquitectura y Protocolos para Redes de Control Distribuido" que se imparte en la titulación de Ingeniero en Informática de la Universidad de Córdoba.

El bus CAN se utiliza ampliamente en el automóvil y en aplicaciones industriales, y se ha estandarizado internacionalmente como ISO 11898 para aplicaciones de alta velocidad (ISO $11898,1993)$ e ISO 11519-2 para aplicaciones de baja velocidad (ISO 11519-2, 1994). Además de esta estandarización ISO/OSI, se tienen otras descripciones CAN 2.0A y CAN 2.0B para los fabricantes de controladores CAN (Bosch, 1991).

Una típica red CAN está formada por varios nodos conectados al bus. Todos los nodos tienen disponible o pueden leer la información que viaja por el bus. La información que circula está compuesta por mensajes. Cada mensaje tiene un identificador que, además de nombrar el mensaje, indica la prioridad de ese mensaje. Ésta se emplea en caso de que dos o más nodos intenten poner mensajes en el bus al mismo tiempo.

El identificador del mensaje debe ser único en la red. La diferencia entre la especificación CAN2.0A y CAN2.0B está en el número de bits del identificador del mensaje. A diferencia de otras redes, este identificador no tiene porqué estar relacionado con el nodo que envía o recibe la información, sino a la información en sí misma. Aunque en la práctica, algunos bits del identificador del mensaje suelen reservarse para asociarlos al nodo que envía la información o al nodo al que va destinada. El formato del mensaje se muestra en la Fig. 1.

\begin{tabular}{|l|l|l|l|l|}
\hline Identificador & Control & Datos & CRC & ACK \\
\hline
\end{tabular}

Fig. 1: Formato de mensaje del bus CAN.

El campo de Control especifica el tipo de trama y el número de bytes de datos que contiene el mensaje. El campo de datos contiene los datos del mensaje pudiendo ser de hasta 8 bytes. El campo CRC se utiliza para detección de errores en la transmisión. CAN define dos valores: bit dominante y bit recesivo. Por definición, el bit dominante sobrescribe al bit recesivo. En la transmisión el nodo que transmite pone el bit ACK al valor recesivo, y en caso de que cualquier nodo del bus haya recibido el mensaje sin errores, deberá poner este bit al valor dominante. Se garantiza con el campo ACK que al menos un nodo ha recibido correctamente el mensaje. Existen además mensajes especiales, que no se detallarán en este trabajo, para informar a todos los nodos del bus que se han producido determinados errores.

La arbitración en el bus se muestra en la Fig. 2. Cuando el bus está inactivo cualquier nodo puede comenzar la transmisión de un mensaje. Si dos o más nodos comienzan la transmisión al mismo tiempo se inicia una arbitración basada en la prioridad del mensaje, es decir de acuerdo a su identificador. Cada nodo lee del bus bit a bit y compara el valor que transmite con el valor que lee en el bus. Si un nodo pone un bit recesivo y lee uno dominante significará que el nodo ha perdido la arbitración. La arbitración en CAN es como una AND global entre nodos. Esta arbitración es no destructiva y el nodo ganador no sufre ninguna interferencia.

Como se puede deducir de la breve exposición de las características del bus CAN (Fig. 2), para la realización de prácticas con el bus CAN se debe tener una red y unos nodos desde los que no solo se pueda transmitir y recibir información sino que se puedan configurar y manipular a bajo nivel. Además en estos nodos debe ejecutarse algún sistema operativo en tiempo real para conseguir unas prácticas lo más próximas a la realidad como sea posible. 


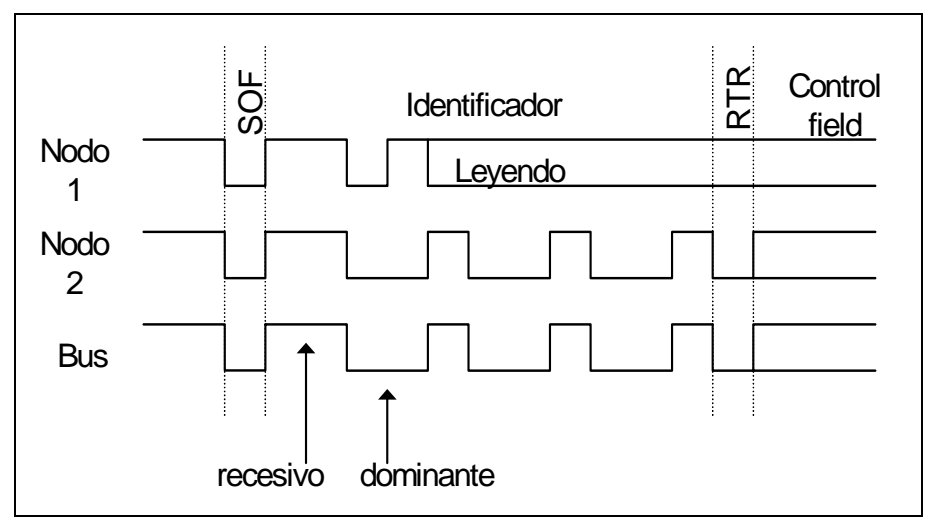

Fig. 2: Arbitración en el bus CAN.

En el mercado se tiene una amplia variedad de placas con interfaz al bus CAN y $\mathrm{PCl}$ (http://www.pcisig.org/), sin embargo, la dificultad que se encuentra reside en que no se dispone de una información detallada del hardware de estas placas por lo que es imposible programar directamente los controladores CAN. Además los fabricantes proporcionan un conjunto de librerías para el manejo de la red CAN solamente para algunos sistemas operativos, siendo difícil encontrar estas librerías para sistemas operativos en tiempo real. Aunque desde el punto de vista industrial, la utilización de estas librerías es la manera más eficiente de trabajo, en el caso de las prácticas, interesa poder trabajar directamente con los registros de los controladores CAN. Así el alumno puede comprender mejor como intercambian información estas redes, que es el objetivo principal de este tipo de prácticas.

Como se ha comentado el objetivo de estas prácticas es conocer el bus CAN, por lo que es necesario trabajar directamente con los controladores y realizar aplicaciones con los sistemas operativos de tiempo real que ya conoce el alumno gracias a otras asignaturas. Teniendo esto en cuenta se pensó que lo más práctico era el desarrollo de una placa con interfaz al bus $\mathrm{CAN}$ y $\mathrm{PCI}$, de forma que se pudiera programar de una manera cómoda y sencilla desde el ordenador personal y con un sistema operativo de tiempo real para dar un mayor realismo a las prácticas.

La utilización de placas comerciales y las funciones de librería que proporcionan, suponiendo que se dispongan de ellas para el sistema operativo de tiempo real utilizado, están pensadas para abstraer el hardware, de forma que el alumno no toma conciencia de la red subyacente. Las operaciones con la red se hacen a través de funciones de alto nivel. En aplicaciones empotradas el entendimiento por parte del alumno de las interacciones software-hardware es esencial (Koopman et al., 2005).

Otras plataformas utilizadas para trabajar directamente con los controladores de bus CAN son la utilización de microcontroladores con controlador CAN integrado en el propio circuito o con controlador CAN independiente, e incluso alternativas que se aproximan aún más al bus, como es la utilización de circuitos lógicos programables que implementan un controlador CAN de propósito general (Mariño et al., 2003).

En los siguientes capítulos se describirán la placa y el conjunto de prácticas que se realizan en la asignatura de "Arquitectura y Protocolos para Redes de Control Distribuido" que se imparte en el segundo curso de la Titulación de $2^{\circ}$ ciclo de I. en Informática. En la descripción de las primeras prácticas se podrá observar las ventajas de disponer de un hardware abierto, que permitirá al alumno interaccionar directamente con el hardware.

\section{CARACTERÍSTICAS DE LA PLACA CAN2PCI}

A la hora de decidir qué características debía tener la placa había que tener en cuenta, además de las prestaciones, su finalidad docente. Por este motivo se optó por utilizar un controlador de bus CAN que fuese flexible y simple de programar. Si el espacio lo permitía debía tener dos canales 
para que de una manera cómoda se tuviese una red CAN de al menos dos nodos por puesto de prácticas. Teniendo en cuenta estas dos premisas se optó por utilizar el controlador SJA1000 (http://www.nxp.com) y montar dos controladores para disponer así de dos canales. Cada controlador trabajaría independientemente. El acceso a los controladores desde el ordenador sería directo, es decir, el programador accedería directamente al controlador sin utilizar ninguna memoria intermedia entre la CPU y el controlador CAN, aunque esta opción podría limitar el rendimiento del nodo CAN. Por otro lado para simplificar el acceso a los controladores se decidió mapear la placa en el espacio de entrada/salida del bus PCl.

Las características principales de la placa CAN2PCI (Fig. 3) son:

- Interfaz al bus CAN: dos canales independientes.

- Controlador de bus CAN SJA1000, con velocidad de hasta 1 MB.

- Posibilidad de utilizar alimentación del bus PCI o alimentación a través del propio bus CAN para el transceiver del bus CAN.

- Aislamiento por optoacopladores del bus CAN.

- Interfaz al bus PCl de 32 bits y 5 Voltios.

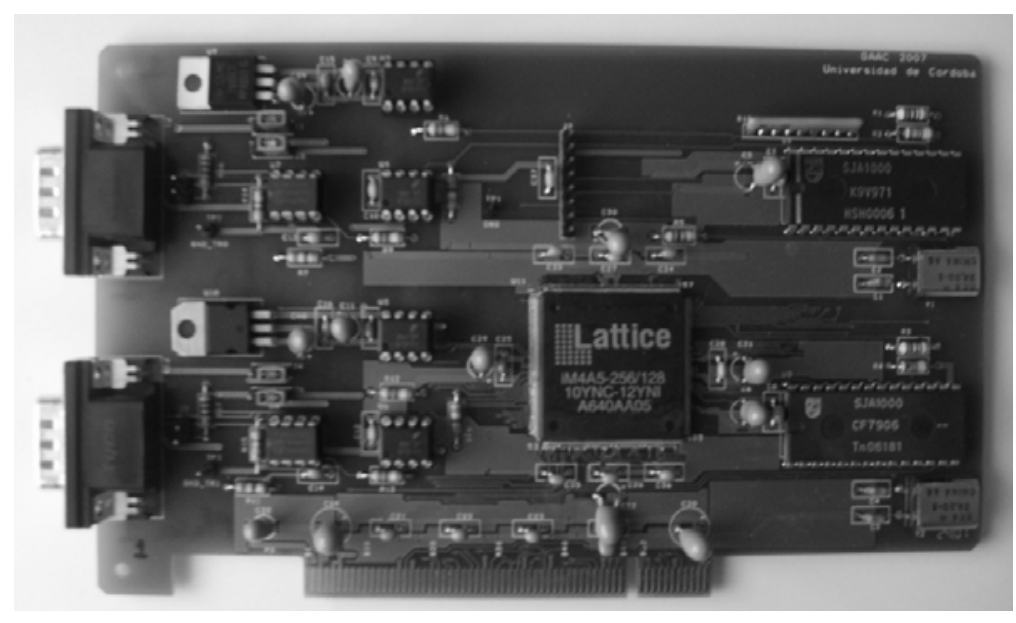

Fig. 3: Fotografía de la placa CAN2PCI.

\section{DESCRIPCIÓN DE LA PLACA CAN2PCI}

La Fig. 4 muestra el diagrama de bloques de la placa CAN2PCI. Toda la lógica de control de esta placa está integrada en el CPLD M4A5-256 de la compañía Lattice Semiconductor (http://www.latticesemi.com). Dicho CPLD realiza el interfaz al bus PCl y genera el bus local de los controladores.

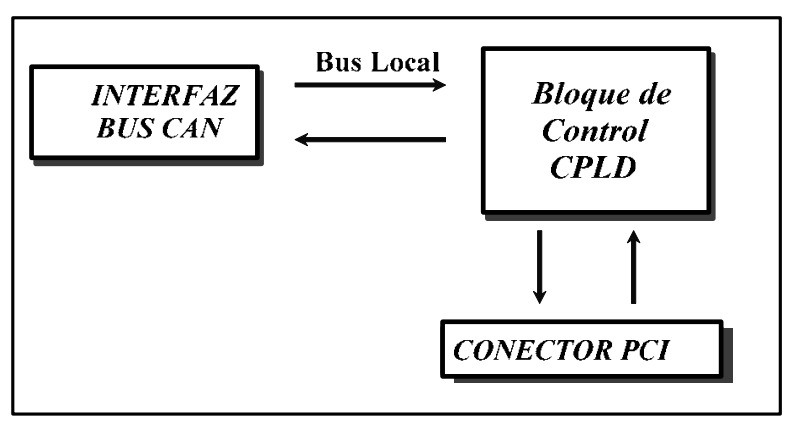

Fig. 4: Diagrama de bloques de la placa CAN2PCI.

La Fig. 5 muestra el diagrama de bloques del interfaz de un canal al bus CAN. Este interfaz está formado por el controlador SJA1000 que se encuentra aislado del transceiver del bus CAN mediante optoacopladores. La alimentación del transceiver se puede seleccionar mediante un puente entre la alimentación del bus $\mathrm{PCI}$ o la alimentación proveniente del bus CAN. 


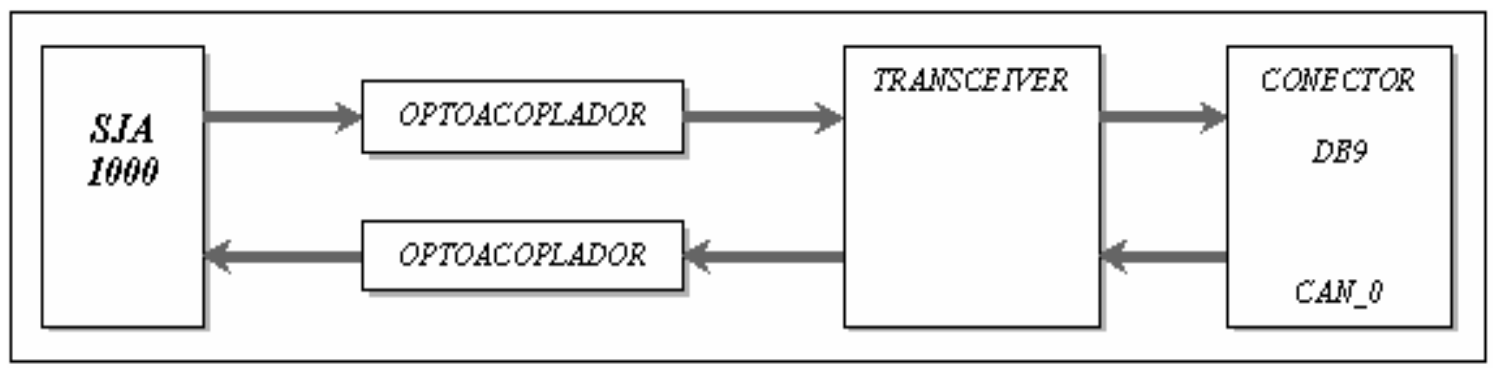

Fig. 5: Interfaz al bus CAN.

\section{PRÁCTICAS EN REDES DE CONTROL UTILIZANDO LA PLACA CAN2PCI}

La placa CAN2PCI está pensada para ser utilizada en la asignatura de "Arquitectura y Protocolos para Redes de Control Distribuido" donde se estudia el bus CAN entre otros buses de control. Se trata de una asignatura optativa de 4,5 créditos cuyo objetivo principal es estudiar los buses de control en los niveles físicos y de enlace, estudiando en profundidad la transmisión de las tramas. Como objetivo secundario se realizan aplicaciones sencillas de control. Para cumplir el objetivo principal de las prácticas, es necesario acceder a los registros del controlador SJA1000 para su configuración y a los buffers de datos de transmisión y recepción. Para el desarrollo de las aplicaciones se utiliza también un sistema operativo en tiempo real, que el alumno ya ha estudiado en otras asignaturas obligatorias, en concreto se utiliza el sistema operativo en tiempo real "Phar Lap ETS" (http://www.ardence.com/embedded).

Las prácticas de la asignatura "Arquitectura y Protocolos para Redes de Control Distribuido" lleva impartiéndose dos cursos y los comentarios que se realizan en este trabajo son fruto de la experiencia adquirida en este tiempo. Aunque el grado de experimentabilidad de esta asignatura permite un máximo de 25 alumnos por grupo de prácticas, hasta el momento, el grupo con mayor número de alumnos ha sido de 14. Las prácticas se realizan en parejas y se utilizaron 7 puestos de prácticas.

Cada puesto de prácticas consta de dos ordenadores personales como muestra la Fig. 6 . Normalmente casi todos los sistemas operativos de tiempo real y en particular el entorno de desarrollo del sistema operativo de tiempo real "Phar Lap ETS", necesitan dos equipos. Uno de ellos, denominado "target", es el equipo donde se ejecutará nuestra aplicación y el otro, denominado "host", contiene el entorno de desarrollo del sistema operativo. Ambos equipos se conectan a través de un canal de comunicación (ethernet, serie o paralelo). En el equipo "host" el usuario escribe el código, lo compila, linka y lo envía para su ejecución al "target", pudiendo además depurarlo. En el equipo "target" estará montada la placa CAN2PCI para la realización de las prácticas.

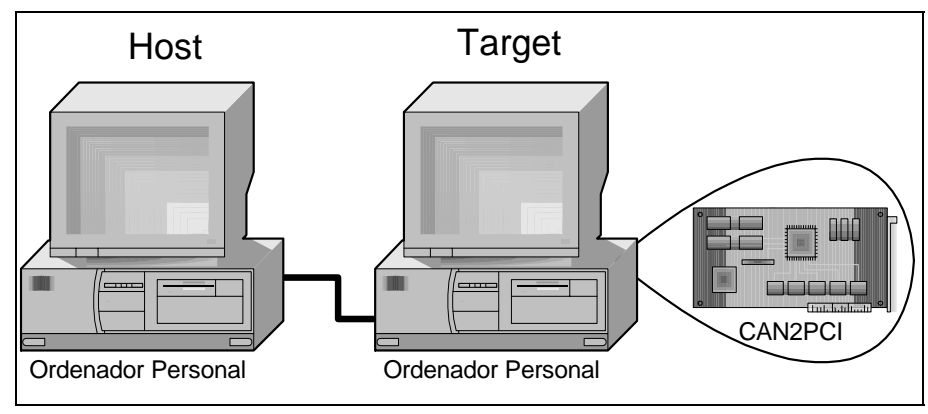

Fig. 6: Puesto de prácticas.

El lenguaje de programación que se utiliza es C. Los alumnos no tienen problemas a la hora de la codificación porque utilizan $C$ habitualmente a lo largo de la carrera y el sistema operativo "Phar Lap ETS" ya lo han utilizado en otras asignaturas. Por tanto el punto de partida es el idóneo para centrarse en los objetivos de las prácticas. 
El objetivo final de las prácticas es realizar una sencilla aplicación parecida al sistema ABS de un automóvil, creando los drivers y librerías para el manejo de la red CAN. La Fig. 7 muestra la estructura de capas de la aplicación.

\begin{tabular}{|c|c|}
\hline Aplicación ABS & \\
\hline $\begin{array}{l}\text { - Colas de transmisión } \\
\text { - Colas de recepción }\end{array}$ & \multirow{2}{*}{ Middleware } \\
\hline $\begin{array}{ll}\text { - } & \text { Envío de tramas } \\
\text { - } & \text { Recepción de tramas } \\
\text { - } & \text { Configuración del controlador CAN } \\
\text { - } & \text { Inicialización del controlador CAN }\end{array}$ & \\
\hline Controlador CAN & \\
\hline
\end{tabular}

Fig. 7: Estructura de capas de la aplicación objetivo de las prácticas.

Las prácticas se han dividido en cuatro bloques y se realizan en sesiones de 2 horas de duración. Se dedican un total de 20 horas a prácticas de las 45 horas totales de la asignatura. A continuación realizaremos una breve descripción de las prácticas.

\section{Transmisión de Tramas CAN}

El objetivo de esta práctica es el estudio de los principales registros del controlador SJA1000 y la transmisión de mensajes por la red. La meta a conseguir es que el alumno transmita mensajes con éxito por la red, por lo que previamente deberá programar la velocidad de transmisión y recepción, y los registros de máscara y aceptación del controlador. Cada puesto de prácticas está aislado del resto y se le conecta un analizador de tramas CAN. En esta práctica se le enseña al alumno a manejar el analizador de tramas CAN y se muestra también en un osciloscopio la trama a nivel físico. Habitualmente la duración de esta práctica es de 2 sesiones de 2 horas.

Estas primeras prácticas son bastante intensas, y aunque se ha comentado anteriormente que la programación en $\mathrm{C}$ no representa ninguna dificultad para los alumnos, sí les resulta dificultoso la tipificación de datos en $\mathrm{C}$. Deben manejar los registros del controlador a nivel de bytes y bits que no son tipos de datos estándar en C. También debe adaptarse a las peculiaridades de la programación en las aplicaciones de control. Los alumnos están acostumbrados a una programación secuencial más que concurrente, y sin embargo, la naturaleza de las aplicaciones de control obliga a pensar en tareas y en muchos casos, tareas que deben realizarse con una cierta periodicidad.

\section{Recepción de Tramas CAN}

El objetivo de esta práctica es la recepción de mensajes. El alumno debe realizar las funciones que le permitan descargar del controlador los mensajes recibidos en el controlador y depositarlos en memoria. En esta práctica los nodos siguen aislados y las tramas se envían desde el analizador que se va conectando a cada puesto. Habitualmente la duración de esta práctica es de 2 horas.

Tanto en la transmisión como en la recepción de los mensajes, se realice por interrupciones o por muestreo, se debe conseguir que el alumno entienda la interacción hardware/software y es en este punto, donde la placa desarrollada y su conocimiento minucioso facilitan la labor docente al profesor y la programación al alumno.

\section{Creación de un Pequeño Middleware}

La tercera práctica consiste en la creación de un pequeño driver o middleware que integra las funciones de transmisión y recepción de mensajes creadas en las dos prácticas anteriores. Al inicio de esta práctica se conectan todos los puestos en red y se comprueba que desde todos los 
puestos se puede transmitir y recibir mensajes correctamente. A continuación se crea una simple aplicación que va lanzando mensajes desde un nodo con identificadores que se incrementan a cada mensaje. En el resto de nodos se crea otra aplicación que va sacando los mensajes del controlador y comprueba que llegan con el identificador esperado. De esta forma se conciencia al alumno de la necesidad de realizar pruebas a las funciones que han implementado.

Para finalizar se crean unas colas de transmisión y recepción que se comprueban utilizando las simples aplicaciones comentadas anteriormente. Se muestra al alumno la necesidad de implementar algún mecanismo de exclusión mutua, ya que las colas son un recurso compartido entre la aplicación de usuario y las rutinas de servicio de interrupción del controlador CAN, encargadas de intercambiar mensajes entre las colas y el controlador CAN. Habitualmente la duración de esta práctica es de 4 horas repartidas en 2 sesiones de 2 horas.

\section{Diseño y Codificación de una Sencilla Aplicación}

Para finalizar las prácticas se desarrolla una pequeña aplicación simulando un sistema "ABS mínimo" de un automóvil. Se crea una red CAN donde cada nodo representa una rueda de un automóvil. Cada nodo genera la velocidad de una rueda de forma simulada y la envía a la red. A su vez recoge de la red la velocidad del resto de las ruedas y del estado del pedal del freno. Un nodo simula el freno que envía la información de su estado a la red. Para simular el bloqueo, la aplicación que se ejecuta en cada nodo genera de forma aleatoria el bloqueo de la rueda enviando velocidad cero. Para comprobar que todo funciona correctamente cada nodo muestra en pantalla del computador la información que envía y recoge de la red y si está disparando el ABS.

La aplicación se descompone en tres tareas que se ejecutan periódicamente y que mantienen una imagen actualizada de las cuatros ruedas y del estado del pedal del freno. Una tarea obtiene la velocidad de la rueda que se simula de manera aleatoria, otra tarea se encarga de transmitir la velocidad de la rueda y recoger los mensajes que llegan por el bus CAN. Por último, una tarea realiza el control del disparo del ABS, comparando la velocidad que tiene su rueda, el resto de ruedas y el estado del freno. La tarea comprueba periódicamente la imagen, y si comprueba que su rueda tiene velocidad cero y se está pisando el freno y alguna del resto de las ruedas tiene una velocidad distinta a cero, dispara el ABS hasta que deje de cumplirse alguna de las condiciones.

Esta última práctica requiere un trabajo de equipo extra, ya que hay que definir los datos que contendrán las tramas que se intercambien, la periodicidad de estas tramas, la velocidad de transmisión, etc. El alumno debe trabajar en programación multihilo, y además esta práctica tiene la complejidad añadida de ser una aplicación distribuida y, por tanto, requiere un esfuerzo de puesta a punto mayor. Habitualmente la duración de esta práctica es de 5 sesiones de 2 horas.

\section{CONCLUSIONES}

El diseño de la placa CAN2PCI con un enfoque docente permite realizar prácticas con el bus CAN a bajo nivel y utilizando un sistema operativo en tiempo real gracias a la posibilidad de tener un hardware "abierto". Esta posibilidad es muy importante en las asignaturas como "Arquitectura y Protocolos para Redes de Control Distribuido", donde el objetivo es el conocimiento de las redes así como de algunos algoritmos distribuidos, y no tanto el desarrollo de aplicaciones de control, que son el cometido de otras asignaturas.

Por otro lado desde el punto de vista del diseño y programación de sistemas empotrados, el que se haya diseñado la placa, nos permite justificar y discutir el diseño hardware, así como, la importancia del conocimiento del interfaz hardware-software para sacar el máximo rendimiento al sistema. En este sentido, se muestra al alumno la importancia del tratamiento de las interrupciones y su interacción con el resto de la aplicación, para mantener la integridad de datos en las colas. Las colas son un recurso compartido entre la aplicación y la rutina de tratamiento de las interrupciones del controlador CAN y debe implementarse la exclusión mutua habilitando y deshabilitando las interrupciones. 
Disponer de dos canales en una sola placa permite tener una red CAN por puesto de prácticas, quedando así aislados los problemas que suelen aparecer en las primeras prácticas. Queda por ultimar y explorar la realización de prácticas de interconexión de redes CAN distintas, para lo que será necesario realizar un puente entre ambas redes.

\section{REFERENCIAS}

Bosch R., CAN specification 2.0, Part A and B. Robert Bosch GmbH, Germany (1991).

ISO 11898, Road vehicles - Interchange of digital information - Controller area network (CAN) for high speed communication (1993).

ISO 11519-2, Road vehicles - Low speed serial data communication - Part 2: Low speed controller area network (CAN) (1994).

Koopman P. y otros doce autores; Undergrate Embedded System Education at Carnegie Mellon, ACM Transactions on Embedded Computing Systems: 4(3), 500-528 (2005).

Mariño P. y otros cinco autores; Development Tools For Industrial Networks Design, Proceedings of the 10th IEEE International Conference on Electronics, Circuits and Systems (2003). 